\title{
Una aproximación a Wikipedia como polisistema cultural
}

\author{
An approach to Wikipedia as a cultural polysystem
}

\author{
Elisa Alonso-Jiménez / elialonso@upo.es \\ Universidad Pablo de Olavide, España
}

\begin{abstract}
This qualitative and interpretative work aims at approaching Wikipedia's cultural facet from a transdisciplinary view. For this purpose, we set to analyse the existing literature that has dealt with Wikipedia in the generic media and in academic publications on the fields of Artificial Intelligence, Information and Education Sciences and Translation Studies as well. In these works - mostly with a descriptive, empirical or pragmatic approachWikipedia is referred to as an online collaborative encyclopaedia that incorporates innovation and content creation devices from the free software movement. Our proposal calls for resorting to theoretical works envisaging Wikipedia's cultural dimension. With a semiotic approach, we have based our analysis hypothesis on the social imaginary proposed by Vázquez Medel, as well as on the Polysystem Theory written by Even-Zohar. We will outline that these authors provide many clues for approaching Wikipedia as a cultural system boasting as a repository of knowledge and human relationships.
\end{abstract}

Key words: Wikipedia, culture, media, information society, free software.

Resumen: En el presente trabajo, de naturaleza cualitativa e interpretativa, se abordan los alcances culturales de Wikipedia desde una aproximación transdisciplinar. Para ello, se analiza la literatura existente centrada en Wikipedia, procedente de medios de comunicación generalistas y de distintas disciplinas académicas (inteligencia artificial, ciencias de la información, educación y estudios de traducción). En dichos trabajos, fundamentalmente de naturaleza descriptiva, empírica y pragmática, se alude a Wikipedia como enciclopedia colaborativa en línea que aúna mecanismos de creación de contenido y de innovación procedentes del software libre. Nuestra propuesta reclama la pertinencia de recurrir a trabajos teóricos que contemplen la dimensión cultural de Wikipedia. Desde una aproximación semiótica, basamos nuestra hipótesis de análisis en el imaginario social de Vázquez Medel y la Teoría de los polisistemas de Even-Zohar, en los que encontramos claves para la consideración de Wikipedia como sistema cultural, en tanto que repositorio de conocimiento y relaciones humanas.

Palabras clave: Wikipedia, cultura, medios, sociedad de la información, software libre. 


\section{Introducción}

En este trabajo se aborda una reflexión crítica, una lectura de los alcances culturales de Wikipedia desde la semiótica y los sistemas culturales (Lotman, 1979, 1996, 1998, 1999, 2000; Even-Zohar, 1990; Vázquez Medel, 1997, 2008/2009). A partir de constructos ya consolidados como la "inteligencia colectiva" propuesto por Lévy (1997) y la "cultura participativa" enunciado por Jenkins et al. (2009), se explora Wikipedia como evocación del discurso multilingüe, cibernético, multimodal y multidireccional que genera nuestra sociedad mundializada. Asimismo, en los siguientes párrafos están latentes consideraciones sobre los conceptos de discurso, sentido, realidad e historia, en torno a los cuales las palabras de Foucault (2010: 74) constituyen un referente ineludible: "El documento no es el instrumento dichoso de una historia que sería en ella misma y con pleno derecho memoria; la historia es el modo peculiar como una sociedad concede estatuto y elabora una masa documental de la que no se separa”. A pesar de que estas consideraciones no son objetivos principales del presente trabajo, se mencionan a modo de apunte en estas líneas introductoras por ser pertinentes para un primer acercamiento a Wikipedia.

En la última década, Wikipedia y sus fundadores, Wales y Sanger, han atraído la atención de los medios de comunicación por distintos motivos, entre otros, por la política editorial de Wikipedia (Waters, 2006; Rajan, 2009), su acceso abierto (de Vrieze, 2012), su neutralidad (Bernstein, 2011) o su fiabilidad (McHenry, 2004). El debate sobre la calidad o precisión de los artículos de Wikipedia o su ámbito temático también ha sido objeto de investigaciones de autores como Giles (2005), Chesney (2006), Gorman (2007) y Halavais y Lackaff (2008).

El espectro de disciplinas que se han interesado por Wikipedia también resulta variado. Así, por ejemplo, encontramos destacables contribuciones desde el ámbito de la inteligencia artificial (Artificial Intelligence, 2013) y desde las ciencias de la información y la educación (Lim, 2009; Head y Eisenberg, 2010; Aibar y Fuster, 2012; Thornton-Verma, 2012, y otros) que han contribuido a una mejor comprensión de Wikipedia, su rol en la sociedad y en la educación, así como al desarrollo de distintas tecnologías que explotan sus posibilidades. Desde los estudios históricos, Burke (2012) ha examinado el surgimiento de Wikipedia como una fuente de conocimiento popular y ha analizado los mecanismos de recuperación de información, siguiendo los postulados de la concepción de la política social y del conocimiento de Foucault. 
Desde las ciencias sociales y las humanidades es posible proponer trabajos cuantitativos y de corte empírico, que ofrecen enormes posibilidades para investigar fenómenos transdisciplinares como Wikipedia. No obstante, consideramos que también tienen cabida propuestas teóricas, como la que aquí presentamos, cuya naturaleza es cualitativa y exploratoria, y persigue examinar la faceta cultural de Wikipedia desde la semiótica y los sistemas culturales.

En primer lugar, se revisa el estado de la cuestión de Wikipedia como enciclopedia en línea, y se examina su relación con el movimiento del software libre, el crowdsourcing y las tecnologías wiki. Posteriormente, se aborda el análisis de Wikipedia bajo la luz de propuestas de corte semiótico ya consolidadas en el ámbito de las ciencias sociales, como la Teoría de los polisistemas enunciada por Even-Zohar (1990) y el imaginario social propuesto por Vázquez Medel (1997, 2008/2009). Así pues, nuestro objetivo específico consiste en determinar en qué medida es posible aplicar ambos marcos conceptuales al caso de Wikipedia, un hecho que, de confirmarse, contribuiría a una mejor comprensión de Wikipedia y serviría de base cualitativa para futuras líneas de investigación en los ámbitos de la comunicación, la sociología, la educación y la traducción, entre otros.

\section{Estado de la cuestión de Wikipedia}

Para contextualizar nuestra propuesta, recurriremos a las reflexiones sobre mundialización, comunicación y nuevo humanismo formuladas por Vázquez Medel en 2002 - una fecha en la que, recordemos, Wikipedia apenas acababa de nacer y era un proyecto absolutamente marginal y desconocido- que, no obstante, parecen aludir a Wikipedia de manera casi profética:

En este nuevo escenario de Babel se hace más urgente que nunca no un idioma que anule a los demás, sino instrumentos de transferencia, de traducción, entre las diferentes lenguas, dialectos e idiolectos. No se trata de construir nuevos territorios que anulen o superen a los otros, sino de establecer espacios de desterritorialización, res nullius, tierras de nadie (o de todos) que puedan ser habitadas sin prevalencias ni imposiciones, experimentadas como lugares de tránsito. Hemos de reconstruir y reconstituir el espacio de lo público en el que se ejercite el sentido profundo de comunidad (Vázquez Medel, 2002: 1).

Como se expone a continuación, a pesar de la corta historia de Wikipedia, existen numerosos trabajos tanto en el ámbito académico como en los medios de divulgación general, que ya se han ocupado de ella. Hay cierto consenso en cuanto a la consideración de Wikipedia como enciclopedia en línea, aunque, 
como se argumentará enseguida, existen algunas características que la diferencian de las enciclopedias tradicionales, como su alcance, nivel de actualización y su modelo de autoría colaborativa.

En las siguientes secciones también se examina la relación de Wikipedia con el movimiento del software libre, del que ha tomado el citado modelo colaborativo, el sistema de revisión de contenido, el patrón de innovación de la cultura y el modelo de licencias de distribución abierta. Por último, dentro de esta sección, destinada a examinar el estado de la cuestión de Wikipedia, se analizan los elementos que configuran su dimensión colaborativa, a saber, la tecnología wiki (como tecnología que hace factible la colaboración desde el punto de vista técnico) y el modelo de producción mediante crowdsourcing (que convierte la posibilidad técnica en una fuerza de trabajo real, gracias a la movilización de voluntarios).

\section{Wikipedia como enciclopedia en línea}

La categorización de Wikipedia bajo el género de las enciclopedias (Stvilia et al., 2005: 444) puede parecer obvio en la actualidad; en parte, porque rápidamente nos hemos acostumbrado a las convenciones de Wikipedia. No obstante, como han apuntado Ayers et al. (2008), Wikipedia difiere de otras enciclopedias tradicionales en varios aspectos, como su alcance más amplio al incluir artículos especializados y generalistas, su dinamismo y alto grado de actualización, su modelo de autoría colaborativa y el hecho de no ser un proyecto comercial:

Wikipedia's scope is far greater than previous encyclopedic projects, however. Encyclopedias have traditionally been published as comprehensive guides to some defined area of knowledge. Wikipedia is instead a collection of both specialist and generalist encyclopedias, linked together into an integrated work. Its articles can be updated immediately: Articles are dynamic, and their content can change from day to day or even (in the case of current events) from minute to minute. Wikipedia's huge scale and rapid updating is possible in part because the authorship model is completely different from earlier projects: The idea of the famous author or expert-written article has been discarded.

Finally, unlike earlier encyclopedias, Wikipedia is a noncommercial project, and its content is deliberately licensed so others can freely use it (Ayers et al., 2008: 37).

Como es sabido, Wikipedia es esencialmente una enciclopedia nacida en Internet, un medio del que hereda una serie de características, como su carácter hipermedia y polisistémico, así como su ubicuidad. Resulta evidente que un proyecto nacido y desarrollado exclusivamente en la web, como 
Wikipedia, no puede ser ajeno a la propia naturaleza del medio donde vive. En el caso de Wikipedia, esta afirmación se constata con contundencia, ya que, más allá de su carácter libre y colaborativo, del que quizá no son siempre conscientes todos sus usuarios, lo que la convierte en un proyecto excepcional son su universalidad y ubicuidad, características derivadas de su medio: Internet. A pesar de que puede resultar redundante apuntarlo, no debemos olvidar que para consultar o editar Wikipedia basta disponer de un ordenador o dispositivo con conexión a Internet.

Su naturaleza hipermedia, construida gracias al wikitexto - una simplificación de los lenguajes de marcado que se emplea en la tecnología wiki- le confiere una dimensión colaborativa y multimodal, en la que se combinan todo tipo de elementos: discurso hipertextual, imágenes, video y sonido. No se trataría, por tanto, de una enciclopedia aislada, sino que estaría vinculada al resto de la web a través de hipervínculos e indexada -y por cierto muy bien ponderada en los logaritmos de búsqueda y recuperación de información-en los buscadores de Internet.

\section{Wikipedia y software libre}

La influencia del movimiento de software libre o de código abierto (FOss, Free/Open Source Software) en Wikipedia resulta evidente, tal como se desprende de las afirmaciones vertidas desde distintas obras inspiradas en FOss. Así, Feller et al. (2005: 486-487) señalan: "From the Open Source movement it [Wikipedia] is considered as a successful application of open source methods and its structural elements to creative production".

Autores como Benkler (2006: 71) han indicado que la incorporación en Wikipedia de algunas de las características procedentes del foss - como su modelo de producción y revisión por pares, y su carácter abierto- han contribuido al éxito de la enciclopedia: "The shift in strategy [of Wikipedia] toward an open, peer-produced model proved enormously successful". Siguiendo esta línea, podemos encontrar numerosos elementos procedentes del movimiento Foss que se han incorporado en Wikipedia y que son además ampliamente visibles. Nos referimos concretamente a su sistema colaborativo de creación de contenido, su modelo de autoría y revisión por pares, la cultura hacker y, por último, a las licencias abiertas de distribución y propiedad intelectual.

Respecto al primero de estos elementos, el aspecto colaborativo, la tecnología wiki y la política editorial de Wikipedia permiten que la creación de 
contenido se lleve a cabo de una determinada forma, especialmente gracias a voluntarios que trabajan de manera colaborativa. A pesar de que cualquier usuario puede editar Wikipedia, existe un núcleo de editores o admins que tienen mayores competencias que el usuario medio y cuyas decisiones ejercen mayor impacto en la comunidad y en los artículos.

Respecto al modelo de autoría y revisión, Wikipedia incorpora diversos mecanismos para promover diferentes tipos de revisión por pares (peer review) que difieren del enfoque tradicional y académico, y siguen las consignas del movimiento foss. Su máxima representación la hallaríamos en el dicho popular formulado por Raymond como la "Ley de Linus" (Linus's Law), la cual defiende que un producto o texto revisado por muchas personas tendrá menos errores: "Given enough eyeballs, all bugs are shallow" (Raymond, 2001: 41). Este posicionamiento ha sido defendido, pero también criticado desde la obra compilada por Feller et al. (2005), ya que, por ejemplo, en el caso de proyectos de software libre no existe garantía de que el código vaya a ser revisado de manera exhaustiva por los miembros de la comunidad, ni tampoco existen evidencias de que la revisión por pares sea más eficaz que los procedimientos de depuración de errores habituales en la industria del software, que normalmente siguen un plan trazado y no obedecen a un impulso aleatorio (Feller et al., 2005: 117, 128, 144, 285). Ciertamente, la revisión por pares en Wikipedia, al igual que sucede en FOss, no tiene por qué ser realizada por un experto o por alguien con experiencia, pues puede ser llevada a cabo virtualmente por cualquiera y obedece a un patrón en gran medida aleatorio. Al respecto, Feller et al. (2005: 252) señalan:

Traditional peer reviews require time for individual study of the code followed by a face-to-face review meeting. These activities must be planned and scheduled, in contrast to the continuous and serendipitous nature of open source peer review.

Las evidentes diferencias que existen entre el modelo tradicional de revisión por pares y la actividad de edición y revisión de Wikipedia, ciertamente azarosa, que se lleva a cabo por parte de los editores de manera voluntaria, los admins, y también por determinados procedimientos automatizados o robots, abren un espacio para la reflexión en torno a la naturaleza de ambas propuestas. A pesar de que dicho análisis no es objetivo de esta contribución, a modo de apunte, esbozaremos aquí la idea de que la revisión en Wikipedia podría obedecer no ya a un modelo de peer-review, sino más bien a un modelo de crowd-review, donde una masa compuesta por agentes humanos y no humanos modelaría Wikipedia con base en un guión no escrito ni preestablecido. 
Además de las tecnologías abiertas descritas en los párrafos anteriores, Wikipedia heredó su esquema de innovación del Foss; nos referimos a la cultura hacker:

Where the general case of applying Open Source methods to other forms of writing has failed, though, there have been some key successes, and there is much to learn from the why and how of such projects. Particularly instructive in this regard is the Wikipedia project (http://wikipedia.org), which brings many of the advantages of modeling culture into a creative enterprise that does not rely on code (Feller et al., 2005: 486).

La cultura hacker, representada por el programador Richard Stallman, tiene como objetivo la libre distribución del código fuente del software, algo que va en contra de los restrictivos contratos de distribución o licencia de la mayoría de los fabricantes y creadores de contenido. Sus orígenes datan de la década de 1980, cuando Stallman lanzó el proyecto GNU con el fin de crear un sistema operativo libre, controlado únicamente por voluntarios, con el respaldo de la Free Software Foundation.

Más allá del impacto del movimiento del foss en la industria del desarrollo de software y tecnologías de la información, como ha señalado Castells (2003), el modo de producción de la cultura hacker instaura un nuevo modelo de innovación que encuentra su razón de ser en la satisfacción personal de quienes contribuyen al proyecto; es decir:

[...] una gran transformación tecnoeconómica necesita un caldo de cultivo en un sistema de valores nuevo que motive a la gente para hacer lo que hace. [...] En la era de la información, la matriz de todo desarrollo (tecnológico, económico, social) está en la innovación, en el valor supremo de la innovación que, potenciada por la revolución tecnológica informacional, incrementa exponencialmente la capacidad de generación de riqueza y de acumulación de poder. Pero innovar no es un valor obvio. Debe estar asociado a una satisfacción personal, del tipo que sea, ligado al acto de la innovación. Eso es la cultura hacker, según Himanen (Castells, 2003: 11-12).

Por último, otra de las características heredadas por Wikipedia del movimiento libre se encuentra en los modelos de distribución y propiedad intelectual por los que se rige, a saber: GNU Free Documentation License y Creative Commons, ambos inspirados en las libertades básicas del movimiento Foss: libertad para usar el software con cualquier fin, cambiar el software para que cada persona lo adapte a sus necesidades, compartir el software y sus modificaciones, así como asegurar que permanecerá como software libre para todos los usuarios (Free Software Foundation y Smith, 2007/2010). 
Wikipedia: wikis and crowdsourcing

La confluencia de elementos que dan lugar a Wikipedia, es decir, tener Internet como medio, la sociedad informacional como contexto y el software libre como estructura y modelo ético, generan lo que Wikipedia es en última instancia: una enciclopedia colaborativa. En esta afirmación constatamos que el elemento diferenciador y novedoso radicaría en una nueva forma de trabajar y generar contenido. Se confirma una vez más la vigencia de las palabras de McLuhan, cuando, adelantándose al impacto que provocaría Internet como nuevo medio o extensión tecnológica en nuestras vidas y en la sociedad, afirmó que "el medio es el mensaje" (McLuhan, 1964/1994: 7).

La plataforma wiki es la que permite esta dimensión colaborativa. La tecnología wiki fue desarrollada por Ward Cunningham en 1995 y adoptada por Wikipedia desde su creación. De hecho, la tecnología wiki es uno de los elementos diferenciadores de Wikipedia respecto al anterior proyecto enciclopédico de Wales, denominado Nupedia (Rodríguez Herrera, 2011: 205206). A diferencia de Nupedia, en la que los artículos una vez escritos eran sometidos a revisión por pares por parte de docentes universitarios y personas expertas, la implementación de la tecnología wiki en Wikipedia configuró una plataforma para un proceso de creación de contenido y revisión de naturaleza colaborativa y simultánea.

No obstante, la tecnología wiki únicamente aporta la posibilidad técnica de colaborar en línea. De lo anterior se desprende que lo que convirtió a Wikipedia en la popular enciclopedia que es hoy fue la voluntad expresada por Wales (y la Wikimedia Foundation) bajo la forma de una llamada pública, donde solicitaban la participación de la gente para un proyecto común. En otras palabras, a pesar de que Wikipedia no se define a sí misma como un proyecto de crowdsourcing, consideramos que contiene muchas de las características atribuidas a este modo de reclutar personal.

En palabras de Howe (2006/2012), quien fue el primero en emplear el término, "crowdsourcing is the act of taking a job traditionally performed by a designated agent (usually an employee) and outsourcing it to an undefined, generally large group of people in the form of an open call". Cabe mencionar que este autor se percató muy pronto de la relación existente entre el crowdsourcing y el software libre, así como del hecho de que ambos elementos convergían en Wikipedia:

The open source software movement proved that network of passionate, geeky volunteers could write code just as well as the highly paid developers at Microsoft of 
Sun Microsystems. [...] Wikipedia showed that the model could be used to create a sprawling and surprisingly comprehensive online encyclopedia (Howe, 2006/2012).

Parece claro que el modelo de crowdsourcing está presente en Wikipedia, desde el momento en que existe una llamada, en este caso, lanzada por Wikimedia Foundation para solicitar la colaboración de voluntarios con el fin de escribir artículos de Wikipedia. Sin embargo, Wikimedia Foundation no sería el único iniciador de los artículos de Wikipedia como actos comunicativos, ya que su función principal consiste en proporcionar un sustento tecnológico y económico al proyecto. Los impulsores de contenido de Wikipedia son, en realidad, cualquier persona que decida crear, modificar, traducir un determinado artículo o, simplemente, solicitar que otras personas lo hagan.

La faceta colaborativa de Wikipedia se refiere a la posibilidad material de que varias personas colaboren en un proyecto común, a la capacidad de editar, crear, revisar o traducir artículos de manera conjunta. La tecnología wiki que subyace en Wikipedia, así como el hecho de que los artículos puedan ser manipulados literalmente por cualquiera, es decir, su carácter abierto, serían los dos elementos definitorios del aspecto colaborativo implícito en Wikipedia.

\section{Wikipedia como sistema cultural}

En una primera aproximación, la consideración de Wikipedia como un polisistema viene determinada en parte por su naturaleza hipermedia y también por su multilingüismo. Cada una de las versiones que existen de Wikipedia en un determinado idioma constituye un sistema que, lejos de estar aislado y ser fijo, se articula en torno a una macroestructura enciclopédica y multilingüe, un proyecto sociocultural dinámico y de carácter abierto. Como señala Saorín (2012: 11), Wikipedia no puede ser considerada como una única enciclopedia, sino como una red de enciclopedias, una por cada idioma, que se encuentran vinculadas entre sí.

En sintonía con esta percepción, existen distintos trabajos en los que se ha examinado la existencia de asimetrías culturales en el contenido y comportamientos diferenciados de las comunidades en las Wikipedias de cada idioma. Así, por ejemplo, en el trabajo empírico de Hara et al. (2010), se detectó la existencia de comportamientos típicos en las discusiones que tienen lugar en Wikipedia en distintos idiomas. En la misma línea, en la investigación de Rinser et al. (2013), se trata de diseñar un sistema eficiente para la 
identificación de grupos de artículos de Wikipedia que describen las mismas entidades del mundo real. Por otro lado, aplicando técnicas de visualización, Biuk-Aghai et al. (2013) han representado mapas de las categorías y temas más colaborativos de Wikipedia en inglés, alemán, chino, sueco y danés.

Asimismo, es oportuno aludir al trabajo de van der Velden (2013), quien explora las posibilidades de descentralizar el diseño de Wikipedia, con el fin de adaptarlo a la producción de contenido indígena. Por último, en el ámbito de la traducción también existen trabajos centrados en el estudio de la faceta cultural de Wikipedia. Así, McDonough (2012), a través de una encuesta online realizada entre traductores voluntarios de Wikipedia, investigó la percepción que tienen respecto a la traducción que se realiza en un entorno de crowdsourcing, concluyendo que su principal motivación es hacer que la información esté disponible para otras personas. Con un enfoque diferente, centrado en explotar las posibilidades de Wikipedia como corpus multilingüe, con vistas, por ejemplo, a la creación de material lexicográfico, también hay numerosas iniciativas (Adafre y de Rijke, 2006; Potthast et al., 2008; Jones et al., 2008; Schönhofen et al., 2008; Adar et al., 2009; Nguyen et al., 2009; Yen et al., 2012; Oliver y Climent, 2012). Estas propuestas ponen de manifiesto la percepción de Wikipedia como fenómeno y producto cultural, así como las variadas posibilidades que ofrece su estudio en el campo de las ciencias sociales y las humanidades.

Como se indicó en la introducción, en este trabajo se reconoce el valor de estudios anteriores y se trata de contribuir a la profundización del conocimiento de la naturaleza de Wikipedia. En un ejercicio que pone a prueba la elasticidad de Wikipedia, enseguida se explora la hipótesis de considerar a Wikipedia como un sistema cultural, aplicando para ello el doble prisma que aportan las teorizaciones ya consolidadas de Even-Zohar (1990) y de Vázquez Medel (1997, 2008/2009). El hecho de que se recurra a estos autores para el análisis no implica en absoluto el rechazo de otras aproximaciones. Como se dijo anteriormente, nuestra propuesta se debe considerar como un punto de partida y, de hecho, reclama la pertinencia de repensar Wikipedia no sólo desde aproximaciones pragmáticas, sino también teóricas.

Así, por ejemplo, en futuros trabajos sería conveniente incorporar una revisión de la concepción de cultura propuesta por Luhmann (1997/2007) en su Teoría de Sistemas Sociales, siguiendo los postulados formulados recientemente por Farías (2014: 86), que contemplan, además de los efectos de los medios de comunicación en la cultura, la importancia de los mediadores materiales en los procesos performativos del mundo. Sin duda, esta vía permitiría contemplar Wikipedia como producto enciclopédico, discurso 
cibernético y polisistema cultural, pero también contemplar la interacción de creadores y consumidores (prosumidores), artefactos tecnológicos y dinámicas sociales que la articulan.

\section{Wikipedia desde el imaginario social de Vázquez Medel}

En 2004, el fundador de Wikipedia, Jimmy Wales, trataba de explicar en qué consistía su proyecto en el transcurso de una entrevista: "Imagine a world in which every single person on the planet is given free access to the sum of all human knowledge. That's what we're doing" (Slashdot, 2004). El hecho de que Wales evoque la metáfora de Wikipedia como "suma del conocimiento humano", como sistema de almacenamiento de información, nos transporta a la teoría lotmaniana de la cultura, así como a los postulados del imaginario social enunciados por Vázquez Medel.

Nuestra hipótesis plantea que Wikipedia puede ser considerada un repositorio de conocimiento humano y, por ende, un sistema cultural, pues uno de los elementos que en opinión de Vázquez Medel domina en la teoría de Lotman es, precisamente, la noción de memoria o la facultad que poseen determinados sistemas de conservar y acumular información.

Lotman (1996: 24) describe la semiosfera en su obra homónima como un gran sistema, "el espacio semiótico fuera del cual es imposible la existencia de la semiosis". En su estudio del citado autor, Cáceres (cit. Lotman, 1996: 261) constata que: "La obra de Iuri Lotman representa, en suma, la búsqueda incesante, desde la coherencia, de una explicación del conjunto de fenómenos culturales y de cada uno de los lenguajes que forman parte de la cultura, de la semiosfera”. Como el propio Lotman afirma (1996: 78), la semiótica de la cultura es la "disciplina que examina la interacción de sistemas semióticos diversamente estructurados, la no uniformidad interna del espacio semiótico, la necesidad del poliglotismo cultural y semiótico"; y más concretamente, expone sobre estas cuestiones:

Desde el punto de vista de la semiótica, la cultura es una inteligencia colectiva y una memoria colectiva, esto es, un mecanismo supraindividual de conservación y transmisión de ciertos comunicados (textos) y de elaboración de otros nuevos. En este sentido, el espacio de la cultura puede ser definido como un espacio de cierta memoria común, esto es, un espacio dentro de cuyos límites algunos textos comunes pueden conservarse y ser actualizados (Lotean, 1996: 157).

Las nociones de lenguaje, cultura y memoria están estrechamente relacionadas en la teorización del fundador de la Escuela de Tartu: 
Todo funcionamiento de un sistema comunicativo supone la existencia de una memoria común de la colectividad. Sin memoria común es imposible tener un lenguaje común. Sin embargo, diversos lenguajes suponen diverso carácter de la memoria. Aquí se trata no sólo de la diferencia del volumen sincrónico de la misma, sino también de su profundidad diacrónica. Podemos formular la tesis: cuanto más complejo es un lenguaje, cuanto más ajustado está para la transmisión y producción de información más compleja, tanto mayor profundidad debe poseer su memoria (Lotman, 1998: 155).

Vázquez Medel (2008/2009: 8) parte de Lotman (1979: 41) para sentar las bases de su "semiótica de la cultura" y la "construcción del imaginario social". En su análisis, observa la repetición de denominaciones existentes en la obra de Lotman para aludir a "cultura": "sistemas comunicativos", "conjunto de la información no genética", "memoria común de la humanidad o de colectivos más restringidos nacionales o sociales”. Además, Vázquez Medel (2008/2009: 8) añade que:

La cultura en tanto que memoria no hereditaria exige, para su propia existencia, otras dos características de extraordinaria importancia: a) su organización sistémica (esta memoria es un sistema: toda cultura necesita, además, unas fronteras sistémicas, se define sobre el fondo de la no-cultura); b) su dimensión comunicacional (cada cultura ha de establecer un sistema de comunicación). Una cultura es, por tanto, memoria, sistema, comunicación. Las variadas dimensiones constructivas de su sistematicidad y los diferentes sistemas de comunicación intra e intercultural serán, pues, dos de los pilares a partir de los cuales se establece una tipología de la cultura.

Wikipedia, además de ajustarse a la categoría de "culturas tecno-comunicacionales, electrónicas, virtualizadas” identificada por Vázquez Medel (2008/2009: 10), cuenta con mecanismos comunicativos y organizativos, fundamentalmente ejecutados a través del debate y el consenso de la comunidad. A modo ilustrativo, mencionaremos tres de estos mecanismos: la autoría colaborativa, los debates que se registran en el historial de cada artículo y el procedimiento para eliminar artículos (Articles for Deletion).

Respecto al modelo de autoría colaborativa, que tantos y tan apasionados debates ha generado, debemos distinguir que hay una tecnología: la tecnología wiki desarrollada en 1995 por Ward Cunningham y adoptada por Wikipedia en 2001, que permite, de facto, la autoría colectiva del contenido de Wikipedia. No obstante, como se ha apuntado en la obra de Ayers et al. (2008), el hecho de que el contenido de Wikipedia pueda ser modificado literalmente por cualquier persona, no significa que la comunidad de editores que sustenta Wikipedia no adopte, como de hecho sucede, numerosas directrices para garantizar, entre otras cuestiones, la verificabilidad de los artículos, que no son producto de investigación original (es decir, que se basan 
en fuentes publicadas), y que adoptan un punto de vista neutral:

When people find out that anyone is allowed to add content to Wikipedia, they often assume that any type of content can be added and in any fashion. But in reality, editing and writing on Wikipedia is constrained by a kaleidoscopic array of rules, or policies [...]. Like a traditional encyclopedia, Wikipedia doesn't accept just anything, although its inclusion policies are clearly much broader that those of most encylopedias. [...] No one in particular has the job of deciding whether an article is suitable for Wikipedia. Rather, contributors submit new pages to the site directly, and they go live immediately without intermediaries. Other contributors then review these articles. Large numbers of new articles are deleted every day, but the new content that conforms to the content policies is kept. [...] These core policies are Verifiability (V), Non Original Research (NOR), and Neutral Point of View (NPOv) (Ayers et al., 2008: 11-12).

Además de la tecnología wiki, Wikipedia posee un modelo de autoría y revisión empleado en el ámbito académico y también en el movimiento del software libre al que hemos hecho referencia con anterioridad. Nos referimos al peer-review o revisión por pares, aunque, como se mencionó, para ser rigurosos deberíamos emplear el término crowd-review o revisión por parte de la masa, con el fin de hacer patente el hecho de que, en principio, diversos agentes humanos y no humanos pueden revisar un determinado contenido de Wikipedia.

El otro mecanismo al que se hizo mención respecto a la organización y comunicación de Wikipedia como sistema cultural, Articles for Deletion, tiene como objetivo garantizar el cumplimiento de las directrices de Wikipedia y se articula en torno a la posibilidad de que un artículo sea propuesto para ser eliminado y que sea la comunidad la que deba alcanzar un consenso, en última instancia, acerca de si se elimina o se mantiene.

A modo ilustrativo, se hace referencia a los trabajos de los investigadores Stefaner et al. (2011), quienes analizaron y visualizaron las 100 discusiones más largas en torno a artículos propuestos para su eliminación, que terminaron con la eliminación del artículo. En su investigación utilizan la metáfora de Wikipedia como un jardín que necesita ser podado, un trabajo del que se encargan muchos jardineros:

Like a garden, an online encyclopedia needs constant weeding. Unlike a garden, an online encyclopedia has thousands of potential gardeners. Over years Wikipedia has developed guidelines and policies to help editors collectively decide whether topics are suitable for inclusion or not. All articles, especially new ones, are reviewed by the community to determine if they meet Wikipedias notability guidelines. Any editor can nominate an article for deletion and, if this nomination is legitimate, a community discussion takes place where any fellow gardeners editors have the opportunity to 
make their voices heard. The usual process is to have a week-long discussion during which community members can discuss in favor or against keeping the article. At the end of this period an administrator reviews the discussion and speaks the final verdict (Stefaner et al., 2011).

Más allá de las directrices y mecanismos que acabamos de mencionar, cabe preguntar si verdaderamente cualquier persona puede escribir en Wikipedia. Como hemos comprobado, técnicamente es posible, por lo que cabría cuestionarse cuál es el perfil de quienes dedican su tiempo a contribuir a la enciclopedia. En contra de lo que se podría pensar, Wattenberg y Viégas (2010: 186-187) descubrieron que no suelen ser personas anónimas, sino usuarios registrados cuyas acciones quedan registradas en su perfil y que, en consecuencia, asumen la responsabilidad de sus actos. También se ha documentado la existencia de editores activos o muy activos que contribuyen enormemente a Wikipedia. Estos usuarios son muy respetados por la comunidad y, en ocasiones, se convierten en admins, es decir, usuarios con capacidad para intervenir de manera más directa en Wikipedia.

A fin de concretar la pertinencia de reclamar la denominación de sistema cultural para Wikipedia recurriremos a su consideración de "realidad dinámica”, tan compleja que parece irreductible, siendo éste uno de los tres principios de todo sistema cultural enunciados por Vázquez Medel (2008/2009: 15). No obstante, entendemos que Wikipedia, en tanto universo semántico donde se conjugan las lenguas naturales y el mundo natural, podría ser una de las vías para alcanzar la meta última de la semiótica cultural postulada por Greimas y Courtés (1979: 100):

El proyecto de una semiótica de la cultura (el de J. Lotman, por ejemplo) está llamado, por consiguiente, a convocar el universo semántico - y, principalmente, sus dos componentes macrosemióticos que son la lengua natural y el mundo natural $-\mathrm{y}$ a tratarlo como una semiótica-objeto a fin de constituir una metasemiótica llamada 'cultura.' [...] Una tarea de este tipo parece exorbitante porque correspondería a la descripción del conjunto de las axiologías, de las ideologías y de las prácticas sociales significantes.

Llegados a este punto, quizá la consideración de Wikipedia como sistema cultural y semiótico convergería con las investigaciones que exploran las posibilidades de la web semántica (Berners-Lee, 1998), un movimiento que persigue superar el estado actual de la web -constituida hoy por datos no estructurados o semiestructurados-, configurando una verdadera web de datos, caracterizada por la posibilidad de intercambiar datos entre todo tipo de sistemas y por la capacidad de que dichos datos sean comprendidos y procesados por máquinas. La potencialidad de Wikipedia como sistema de sistemas culturales abiertos, tecnocomunicacionales y virtualizados, capaz de 
conservar información y mantener un registro de las interacciones y comunicaciones entre los distintos agentes, de las decisiones basadas en el consenso dentro de su seno, se postula como un recurso que podría ser un catalizador de esa nueva dimensión sociocomunicacional de la web.

\section{Wikipedia y la Teoria de los polisistemas de Even-Zohar}

Ante las observaciones que ya hemos apuntado en torno a la naturaleza de Wikipedia y sus características culturales, resulta difícil resistirse a la idea de utilizar la metáfora del polisistema aplicada a Wikipedia. Por este motivo, esa es otra de nuestras hipótesis. Seguidamente, se analiza la Teoría de los polisistemas y se examina en qué medida podemos mantener o rechazar nuestra hipótesis.

Desde la semiótica se reconoce a Even-Zohar como "autor de una de las teorías literaturológicas y culturológicas más programáticamente sistémicas y autor, asimismo, profundamente influido por la semiótica eslava (y, naturalmente, por la semiótica de la Escuela de Tartu-Moscú) (Lampis, 2009/2010: 36).

Even-Zohar desarrolló su Teoría de los Polisistemas desde 1969 hasta 2011. No obstante, podemos considerar que sentó las bases de su hipótesis en 1990, en una edición especial de Poetics Today (Even-Zohar 1990), donde reconoce la gran contribución realizada desde el funcionalismo moderno, al introducir la noción del sistema, superando así la consideración de los fenómenos semióticos como meros conglomerados de elementos dispares (Even-Zohar, 2007/2011: 1).

Gracias a ello considera que "el funcionalismo ha alterado profundamente tanto las estructuras como los métodos, las preguntas y las respuestas de todas las disciplinas en que se ha introducido" (ibidem). Even-Zohar lamenta que los dos programas existentes dentro del enfoque funcional, a saber, el estático (emanado de la escuela de Ginebra) y el dinámico (abanderado por los Formalistas rusos y los Estructuralistas checos, y que él mismo adopta), no hayan sabido conjugarse mejor y haya prevalecido -especialmente en lingüística y en la teoría de la literatura- el enfoque estático (Even-Zohar, 2007/2011: 2). Su posicionamiento al acuñar el término polisistema es más que una mera convención terminológica:

Su propósito es hacer explícita una concepción del sistema como algo dinámico y heterogéneo, opuesta al enfoque sincronístico. De este modo, enfatiza la multiplicidad de intersecciones y, de ahí, la mayor complejidad en la estructuración que ello implica. 
Recalca además que, para que un sistema funcione, no es necesario postular su uniformidad (Even-Zohar, 2007/2011: 3-4).

Even-Zohar (2007/2010: 3) considera que tanto la sincronía como la diacronía son históricas. Sin embargo, entiende que la sincronía no puede ni debe identificarse con lo estático, ya que, en un momento dado, puede haber más de un complejo diacrónico en un eje:

Así pues, por una parte, un sistema sincrónico se compone de sincronía y diacronía; por otra, cada una de ellas por separado es obviamente un sistema. En segundo lugar, si las ideas de estructuración y sistemicidad ya no necesitan identificarse con la homogeneidad, un sistema semiótico puede concebirse como una estructura heterogénea y abierta. Rara vez es, por tanto, un monosistema, sino que se trata necesariamente de un polisistema: un sistema múltiple, un sistema de varios sistemas con intersecciones y superposiciones mutuas, que usa diferentes opciones concurrentes, pero que funciona como un único todo estructurado, cuyos miembros son interdependientes.

Desde nuestra óptica, el carácter dinámico de Wikipedia se ajusta a esta característica del polisistema, al ser un repositorio de información que cambia a lo largo del tiempo pero, a la vez, mantiene un registro de cada uno de sus estados sincrónicos a modo de foto fija de un instante, de anillo de crecimiento en el árbol del conocimiento humano. También, su naturaleza heterogénea y diversa, surgida de su faceta colaborativa y plurisubjetiva, permite mantener la hipótesis inicial. Even-Zohar (2007/2010: 4) recalca que el polisistema abarca las nociones de sistema cerrado y de sistema abierto, es decir, "tanto la idea de un conjunto-de-relaciones cerrado, donde los miembros reciben su valor de sus respectivas oposiciones, como la idea de una estructura abierta que consiste en varias redes-de-relaciones de este tipo que concurren".

Siguiendo nuestra argumentación, Wikipedia como enciclopedia, como discurso que se puede leer y escribir, hace gala de un carácter ciertamente abierto; tanto, que ha sido considerada en algunos momentos como subversiva. No obstante, las propias directrices de Wikipedia (Punto de Vista Neutral, Verificabilidad, Investigación No Original, Articles for Deletion, etc.) permiten a la comunidad ejercer un control férreo sobre la enciclopedia. Además, esta comunidad de individuos establecen variadas relaciones que no siempre son de igual a igual, sino que podríamos considerar que existe una oligarquía compuesta por los admins, que gracias a sus especiales capacidades $\mathrm{y}$ a su prestigio pueden ejercer una posición dominante en el sistema.

Otra de las características innovadoras de la Teoría de los Polisistemas es su integración de elementos heterogéneos dentro del polisistema y la incorporación de una visión dinámica del mismo, impulsada por las tensiones 
existentes entre diferentes estratos, así como los movimientos entre centros y periferias:

La heterogeneidad puede reconciliarse con la funcionalidad si asumimos que las unidades (elementos o funciones) que aparentemente son irreconciliables, más que correlacionarse las unas con las otras en tanto que unidades (elementos o funciones) individuales, constituyen sistemas de opciones concurrentes parcialmente alternativos. Estos sistemas no son iguales, sino que están jerarquizados en el seno del polisistema. Lo que constituye el estado sincrónico (dinámico) del sistema — ha sugerido Tynjanov— es la lucha permanente entre varios estratos. Lo que constituye el cambio en el eje diacrónico es la victoria de un estrato sobre otro. En este movimiento opuestamente centrífugo y centrípeto, los fenómenos son arrastrados del centro a la periferia, mientras, en sentido contrario, ciertos fenómenos pueden abrirse paso hasta el centro y ocuparlo (Even-Zohar, 2007/2010: 5-6).

Esta noción de polisistema donde existen tensiones entre centros y periferias ha dado lugar a la aplicación de la teoría de Even-Zohar al ámbito de la traducción literaria. Ciertamente, es una de las propuestas más fecundas de las últimas décadas en este campo. No es de extrañar, si tenemos en cuenta que refleja a la perfección la existencia de sistemas literarios en distintos idiomas sujetos a tensiones (intra-relaciones, relaciones dentro del sistema, o inter-relaciones, relaciones entre varios sistemas).

El fenómeno mismo de Wikipedia, probablemente, puede considerarse periférico, al menos en sus inicios, ya que poco a poco, gracias a las contribuciones de muchas personas, avanza hacia el centro del macrosistema. Asumiendo la paradoja del origen periférico de Wikipedia, en tanto que polisistema, también tiene sus propios centros y periferias. En el centro del polisistema se encontraría en la actualidad la Wikipedia en inglés, mientras que en la periferia se hallarían las Wikipedias en otros idiomas. Es innegable que existen tensiones entre centro y periferia, que probablemente se plasman en traducciones, adaptaciones o enriquecimiento mutuo entre los sistemas y los individuos de la comunidad. Resulta muy versátil aplicar la metáfora del polisistema en Wikipedia, ya que evoca de manera sugerente la idea de distintos sistemas (una Wikipedia por cada idioma) que están intra e inter-relacionados. Cada una de las Wikipedias puede tener su propia morfología gnoseológica, de manera que la estructura de sus categorías de conocimiento no sería simétrica ni igual en todas ellas.

En un segundo nivel de análisis, la aplicabilidad de los conceptos construidos por Even-Zohar (2007/2010: 7 y 10) al estudio de las literaturas nacionales, como es el caso de "canon" en el sentido de normas y obras literarias aceptadas como legítimas por los círculos dominantes y de "repertorio" 
como agregado de leyes y elementos que rigen la producción de textos, ha contribuido también a la popularidad de la Teoría de los Polisistemas en el ámbito de la traducción literaria. En este sentido, los artículos de Wikipedia no serían un repertorio en sí mismo, sino manifestaciones parciales del polisistema. De la misma forma, en el sistema de Wikipedia los artículos no desempeñarían un papel en los procesos de canonización, sino que serían el resultado de esos procesos.

\section{Conclusiones}

Consideramos que un sistema tan complejo como Wikipedia requiere de una visión transdisciplinar para su análisis semiótico y cultural, a la que hemos tratado de contribuir desde estas líneas, con el objetivo adicional de suscitar nuevos enfoques y líneas de investigación desde una plétora de aproximaciones, entre las que se encontrarían la tecnologías de la información, la comunicación, la sociología y la traducción.

En nuestro interés por examinar los alcances culturales de Wikipedia, observamos que en su relativamente corta trayectoria y debido a su excepcionalidad ha recibido la atención tanto de los medios de comunicación, como de distintas disciplinas, como la inteligencia artificial, las ciencias de la información, la educación y los estudios de traducción. Asimismo, se revisaron los trabajos descriptivos sobre Wikipedia existentes hasta la fecha, que la definen como enciclopedia en línea, afín con la filosofía del software libre y de proyecto colaborativo.

Respecto a la primera de las aproximaciones, Wikipedia comparte con otras enciclopedias tradicionales una base común, pero difiere en otros aspectos: posee un alcance más amplio, incluye artículos especializados y generalistas, posee un mayor dinamismo, se actualiza con gran frecuencia, se articula en torno a un modelo de autoría colaborativa y no es un proyecto comercial. Además, por tratarse de una enciclopedia nacida en Internet ha tomado una serie de características de ese medio (carácter hipermedia, polisistémico y ubicuo). Así, la tecnología wiki que le sirve de sustento contribuye a su dimensión colaborativa y multimodal.

En cuanto a la segunda aproximación, como se apuntó, entre los elementos que vinculan Wikipedia con el movimiento del software libre se encuentran su sistema colaborativo de creación de contenido, su modelo de autoría y revisión por pares, la cultura hacker y, por último, las licencias abiertas de distribución y propiedad intelectual que la regulan. De acuerdo con lo que se 
argumentó, el modelo de revisión de contenido en Wikipedia obedecería no ya a un sistema de revisión por pares o peer-review, sino más bien a un modelo de crowd-review, en el que intervendría una masa compuesta por agentes humanos y no humanos.

En tercer lugar, Wikipedia, como proyecto colaborativo, fue la primera enciclopedia en la que se impuso como elemento diferenciador una nueva forma de trabajar y generar contenido. Como se expuso, la tecnología wiki es la que permite la posibilidad técnica de trabajar (redactar, revisar, traducir y acordar directivas) de manera colaborativa, mientras que el crowdsourcing sería el mecanismo para recabar la participación de numerosas personas.

En resumen, consideramos que las bases de Wikipedia se asientan en los mismos cimientos que la sociedad informacional y constituyen una amalgama de presupuestos éticos, modelos de innovación, tecnologías de lectoescritura colaborativas y nuevos paradigmas culturales. La consecuencia de todo ello es un proyecto sólido y abierto, un repositorio multilingüe de conocimiento y de relaciones humanas.

En cuanto a la hipótesis principal sobre la posibilidad de que Wikipedia sea considerada un sistema cultural en los sentidos propuestos desde la semiótica por Lotman, Vázquez Medel y Even-Zohar, hemos tratado de apuntar una serie de consideraciones. En particular, la selección de estos autores en particular no pretende ser excluyente ni restrictiva y así lo expresamos en nuestra intención de continuar explorando esta línea de trabajo, mediante la inclusión de autores como Luhmann, así como de otros estudios semióticos, que aportarían valiosos puntos de vista.

En este análisis, se constató la existencia de trabajos previos, fundamentalmente de corte empírico, que se aproximan a Wikipedia escrutando su dimensión cultural y sus posibilidades como corpus multilingüe. No obstante, se encontró cierta tendencia al pragmatismo en algunos estudios centrados en Wikipedia, que consideramos pueden ser complementados o incluso sustentados con trabajos teóricos consolidados como los de Lotman, Vázquez Medel y Even-Zohar.

El imaginario social de Vázquez Medel, que entronca con la teoría lotmaniana de la cultura, se empleó en este trabajo para explorar la posibilidad de considerar Wikipedia como un sistema cultural, en cuanto que memoria no hereditaria, sistémica y comunicacional. Como hemos esgrimido, Wikipedia responde a los cánones descritos desde la semiótica para los sistemas culturales, ya que posee mecanismos comunicativos ejecutados a través del debate y el consenso de la comunidad, como, por ejemplo, la autoría 
colaborativa (sustentada por la tecnología wiki y la revisión que hemos denominado crowd-review), los debates y el procedimiento para la eliminación de artículos.

En este análisis se esbozó también una futura vía de investigación, a partir del concepto de "semiótica de la cultura" que consideramos hace converger la posibilidad de convocar el universo semántico (compuesto por la lengua natural y el mundo natural), que es objeto de estudio de la semiótica, con las posibilidades que suscita la investigación en web semántica.

La Teoría de los polisistemas enunciada por Even-Zohar define una serie de características que debe tener todo polisistema, como el enfoque sincronístico y la multiplicidad de intersecciones, abarcando las nociones de sistema cerrado y abierto. Otra de las peculiaridades que, según EvenZohar, poseen los polisistemas es la integración de elementos no uniformes o heterogéneos, así como una visión dinámica impulsada por tensiones entre diferentes estratos, y entre centros y periferias. De acuerdo con lo aquí argumentado, Wikipedia es un sistema abierto y dinámico, en tanto que se perfila como un repositorio de información que cambia a lo largo del tiempo, pero que, gracias a la tecnología wiki, mantiene un historial de cada uno de sus estados. Su faceta colaborativa y plurisubjetiva permite, de facto, la existencia de elementos heterogéneos, pero, al mismo tiempo, las directrices que se han desarrollado de manera consensuada garantizan en gran medida el control por parte de la comunidad y sus editores más expertos.

Wikipedia, como discurso, reúne los componentes de una transmodernidad diversa y plurisubjetiva, y articula varios sistemas (una Wikipedia por cada idioma) con sus propios centros y periferias, tanto en lo que respecta a su temática, como en el uso o predilección por uno u otro idioma. Así, la dimensión multilingüe de Wikipedia genera un macrosistema en cuyo centro se sitúa actualmente la Wikipedia en inglés, rodeada por el resto de los idiomas que se extienden en mayor o menor medida hacia la periferia, en función de circunstancias como su peso demográfico, políticas lingüísticas o activismo de sus comunidades. Por último, observamos que Wikipedia, que nació originariamente como proyecto periférico, poco a poco va adquiriendo una posición central y en la actualidad recibe gran atención por parte de los medios de comunicación y la academia.

Del ejercicio de análisis, comparación e interpretación de carácter cualitativo que pretende ser esta contribución - y retomando nuestra hipótesis inicial, es decir, si es posible analizar Wikipedia bajo las propuestas semióticas de Lotman, Vázquez Medel y de Even-Zohar-, se concluye que es plausible considerar Wikipedia como un polisistema social y cultural, multilin- 
güe y multimodal, cuyos discursos consensuados y colaborativos son a la vez reflejo y creación de la realidad, y que nos ofrece la clave, a modo de Piedra Rosetta, para un eventual redescubrimiento de lo humano, sus discursos y sus significados.

\section{Bibliografía}

Adafre, Sisay F. y Maarten de Rijke, M. (2006), "Finding similar sentences across multiple languages in Wikipedia", en Proceedings of the Workshop on NEW TEXT Wikis and Blogs and Other Dynamic Text Sources, 11 th Conference of the European Chapter of the Association for Computational Linguistics, EACL-2006, Trento, Italia.

Adar, Eytan, Michael Skinner y Daniel S. Weld (2009), "Information arbitrage across multilingual Wikipedia", en Proceedings of the Second ACM International Conference on Web Search and Data Mining, Nueva York, EUA.

Ayers, Phoebe, Charles Matthews y Ben Yates (2008), How Wikipedia works: And how you can be a part of it, San Francisco: No Starch Press.

Benkler, Yochai (2006), The wealth of networks. How social production transforms markets and freedom, New Haven/Londres: Yale University Press.

Biuk-Aghai, Robert P., Pang, Cheong-Iao y Si, Yain-Whar (2013), "Visualizing large-scale human collaboration in Wikipedia”, en Future Generation Computer System, vol. 31. Amsterdam: Elsevier.

Burke, Peter (2012), A Social History of Knowledge, ii: From the Encyclopédie to Wikipedia, Cambridge/Malden: Polity Press.

Castells, Manuel (2003), "Internet, libertad y sociedad: Una perspectiva analítica”, en Polis, Revista Académica de la Universidad Bolivariana, vol. 1, núm. 4, Santiago, Chile: Cispo (Centro de Investigación sobre Sociedad y Políticas Públicas).

Even-Zohar, Itamar (1990), “Polysystem Studies”, en Poetics Today, vol. 11, núm. 1. Durham: Duke University Press.

Even-Zohar, Itamar (2007/2011), Polisistemas de cultura, Tel Aviv: Universidad de Tel Aviv, Laboratorio de Investigación de la Cultura.

Feller, Joseph, Brian Fitzgerald, Schott A. Hissam y Karim R. Lakhani [eds.] (2005), Perspectives on Free and Open Source Software, Cambridge/Londres: The MIT Press.

Foucault, Michael (2010), La arqueología del saber, México: Siglo Xxi Editores.

Hovy, Eduard, Navigli, Roberto, Ponzetto, Simeone Paolo (eds.) (2013), "Artificial Intelligence, Wikipedia and Semi-Structured Resources" (número monográfico especial), en Artificial Intelligence, vol. 194, Amsterdam: Elsevier.

Jenkins, Henry et al. (2009), Confronting the challenges of participatory culture, Cambridge/ London: The MIT Press.

Jones, Gareth J.F. et al. (2008), "Domain-specific query translation for multilingual information access using machine translation augmented with dictionaries mined from Wikipedia”, en CLIA 2008 - 2nd International Workshop on Cross Lingual Information Access: Addressing the Information Need of Multilingual Societies, Hyderabad, India. 
Convergencia Revista de Ciencias Sociales, núm. 68, 2015, Universidad Autónoma del Estado de México

Lévy, Pierre (1997), Collective Intelligence: Mankind's Emerging World in Cyberspace, Cambridge, EUA: Perseus Publishing.

Lotman, Iuri M. y Escuela de Tartu (1979), Semiótica de la cultura, Madrid: Cátedra.

Lotman, Iuri M. (1996), La semiosfera I. Semiótica de la cultura y del texto, Madrid: Cátedra.

Lotman, Iuri M. (1998), La semiosfera II. Semiótica de la cultura, del texto, de la conducta y del espacio, Madrid: Cátedra.

Lotman, Iuri M. (1999), Cultura y explosión, Barcelona: Gedisa.

Lotman, Iuri M. (2000), La semiosfera III. Semiótica de las artes y de la cultura, Madrid: Cátedra.

Luhmann, Niklas (1997/2007), La Sociedad de la Sociedad, México, DF: Herder.

McDonough Dolmaya, Julie (2012), "Analyzing the Crowdsourcing Model and Its Impact on Public Perceptions of Translation”, en The Translator, vol. 18, núm. 2, Manchester: St. Jerome Publishing.

McLuhan, Marshall (1964/1994), Understanding Media. The Extensions of Man, Cambridge/Londres: The MIT Press.

Nguyen, D. et al. (2009), "WikiTranslate: Query translation for cross-lingual information retrieval using only Wikipedia”, en Evaluating Systems for Multilingual and Multimodal Information Access. 9th Workshop of the Cross_Language Evaluation Forum, CLEF 2008, Aarhus, Dinamarca.

Oliver, Antoni y Salvador Climent (2012), “Using Wikipedia to develop language resources: WordNet 3.0 in Catalan and Spanish”, en Digithum, núm. 14, Barcelona: Universitat Oberta de Catalunya.

Potthast, Martin, Benno Stein y Maik Anderka (2008), “A Wikipedia-based multilingual retrieval model”, en Macdonald, C. et al. [eds.], Advances in Information Retrieval. 30th European Conference on IR Research, ECIR 2008, Glasgow: Springer.

Raymond, Eric S. (2001), The cathedral and the bazaar, Sebastopol: O'Reilly.

Rinser, Daniel, Dustin Lange, Felix Naumann (2013), "Cross-lingual entity matching and infobox alignment in Wikipedia”, en Information Systems, vol. 38, núm 6, Oxford: Elsevier.

Rodríguez Herrera, Daniel (2011), Ceros y unos: La increíble historia de la informática, Internet y los videojuegos, Madrid: Ciudadela Libros.

Saorín, Tomás (2012), Wikipedia de la $A$ a la $W$, Barcelona: Uoc.

Schönhofen, Péter et al. (2008), "Cross-language retrieval with Wikipedia”, en Advances in Multilingual and Multimodal Information Retrieval, 8th Workshop on the CrossLanguage Evaluation Forum, CLEF 2007, Budapest, Hungría.

Van der Velden, Maja (2013), "Decentering Design: Wikipedia and Indigenous Knowledge”, en International Journal of Human-Computer Interaction, vol. 29, núm. 4, Philadelphia: Taylor \& Francis.

Vázquez Medel, Manuel Ángel (1997), "La semiótica de la cultura y la construcción del imaginario social”, en Cáceres Sánchez, Manuel [ed.], En la esfera semiótica lotmaniana, Valencia: Episteme. 
Wattenberg, Martin y Fernanda Viégas (2010), "Beautiful history: Visualizing Wikipedia”, en Steele, Julie y Noah Iliinsky [eds.], Beautiful visualization, Sebastopol: O’Reilly.

Ye, Zheng et al. (2012), "Mining a Multilingual Association Dictionary From Wikipedia for Cross-Language Information Retrieval”, en Journal of the American Society for Information Science and Technology, vol. 63, núm. 12. Hoboken, EUA: Wiley-Blackwell.

\section{Recursos electrónicos}

Aibar, Eduard y Mayo Fuster [eds.] (2012), “Academic research into Wikipedia”, en Digithum, núm. 14. Disponible en: http://journals.uoc.edu/ojs/index.php/digithum/ article/view/n14-aibar-fuster/n14-aibar-fuster-eng [25 de enero de 2013].

Berners-Lee, Tim (1998), Semantic Web Road map. Disponible en: http://www.w3.org/ DesignIssues/Semantic.html [25 de enero de 2013].

Bernstein, Jon (3 de febrero de 2011), “Wikipedia's benevolent dictator”, en NewStatesman. Disponible en: http://www.newstatesman.com/digital/2011/01/jimmy-waleswikipedia-site [25 de enero de 2013].

Chesney, Thomas (2006), “An empirical examination of Wikipedia's credibility”, en First Monday, vol. 11, núm. 11. Disponible en: http://firstmonday.org/article/ view/1413/1331 [25 de enero de 2013].

de Vrieze, Jop (2 de mayo de 2012), "Government Enlists Wikipedia Founder for Open Access Policy”, en Science Insider. Disponible en: http://news.sciencemag. org/2012/05/u.k.-government-enlists-wikipedia-founder-open-access-policy [25 de enero de 2013].

Farías, Ignacio (2014), "Cultura: la performación de mundos sociomateriales", en Convergencia. Revista de Ciencias Sociales, vol. 21, núm. 64. Disponible en: http:// www.redalyc.org/articulo.oa?id=10529071003 [25 de enero de 2013].

Free Software Foundation y Brett Smith, B. (2007/2010), A Quick Guide to GPLv3. Disponible en: http://www.gnu.org/licenses/quick-guide-gplv3.html [25 de enero de 2013].

Giles, Jim (2005), "Internet encyclopaedias go head to head", en Nature, núm. 428. Disponible en: http://www.u.arizona.edu/ trevors/nature_15dec2005_wikipedia. pdf [25 de enero de 2013].

Gorman, G.E. (2007), "A tale of information ethics and encyclopaedias; or, is Wikipedia just another Internet scam?”, en Online Information Review, vol. 31, núm. 3. Disponible en: http://www.deepdyve.com/lp/emerald-publishing/a-tale-of-information-ethics-andencyclop-dias-or-is-wikipedia-just-QUQI27reYj [25 de enero de 2013].

Halavais, Alexander y Derek Lackaff (2008), "An analysis of topical coverage of Wikipedia”, en Journal of Computer-Mediated Communication, núm. 13. Disponible en: http:// onlinelibrary.wiley.com/doi/10.1111/j.1083-6101.2008.00403.x/full [25 de enero de 2013].

Hara, Noriko, Pnina Schachaf, Khe F. Hew (2010), "Cross-Cultural Analysis of the Wikipedia Community", en Journal of the American Society for Information Science and Technology, vol. 61, núm. 10. Disponible en: http://eprints.rclis.org/15529/1/ 
Convergencia Revista de Ciencias Sociales, núm. 68, 2015, Universidad Autónoma del Estado de México

WikipediaCommunity.pdf [25 de enero de 2013].

Head, Alison J. y Michael B. Eisenberg (2010), "How today's college students use Wikipedia for course related research”, en First Monday, vol. 15, núm. 3. Disponible en: http:// firstmonday.org/article/view/2830/2476 [25 de enero de 2013].

Howe, Jeff (2006/2012), "Crowdsourcing. Why the Power of the Crowd is Driving the Future of Business" (Blog). Disponible en: http://www.crowdsourcing.com/ [25 de enero de 2013].

Lampis, Mirko (2009/2010), "La semiótica de la cultura; hacia una modelización sistemática de los procesos semióticos”, en Entretextos, 14/15/16. Disponible en: http://www. ugr.es/ mcaceres/entretextos/entre14-16/pdf/entretextos14-16.pdf [25 de enero de 2013].

Lim, Sook (2009), "How and why do college students use Wikipedia?", en Journal of the American Society for Information Science and Technology, vol. 60, núm. 11. Disponible en: http://onlinelibrary.wiley.com/doi/10.1002/asi.21142/abstract [25 de enero de 2013].

McHenry, Robert (15 de noviembre de 2004), “The Faith-Based Encyclopedia”, en TCS Daily. Disponible en: http://www.ideasinactiontv.com/tcs_daily/2004/11/the-faithbased-encyclopedia.html [25 de enero de 2013].

Rajan, Amol (27 de agosto de 2009), “The Big Question: Why has Wikipedia changed editorial policy, and will it improve the website?", en The Independent. Disponible en: http:// www.independent.co.uk/life-style/gadgets-and-tech/features/the-big-question-whyhas-wikipedia-changed-editorial-policy-and-will-it-improve-the-website-1777569. html [25 de enero de 2013].

Slashdot (28 de julio de 2004), “Wikipedia founder Jimmy Wales responds". Disponible en: http://slashdot.org/story/04/07/28/1351230/wikipedia-founder-jimmy-walesresponds [25 de enero de 2013].

Stefaner, Moritz, Dario Taraborelli, Giovani L. Ciampaglia (2011), Notabilia - Visualizing Deletion Discussions on Wikipedia. Disponible en: http://notabilia.net [25 de enero de 2013].

Stvilia, Besiki et al. (2005), "Assessing information quality of a community-based encyclopedia", en Proceedings of the International Conference on Information QualityICIQ 2005. Disponible en: http://mailer.fsu.edu/ bstvilia/papers/quantWiki.pdf [25 de enero de 2013].

Thornton-Verma, Henrietta (18 de abril de 2012), "Reaching the Wikipedia Generation", en Library Journal. Disponible en: http://lj.libraryjournal.com/2012/04/publishing/ reaching-the-wikipedia-generation-lj-recently-gathered-publishers-aggregators-andlibrarians-to-discuss-trends-and-thorny-issues-in-reference/\#_ [25 de enero de 2013].

Vázquez Medel, Manuel Ángel (2002), "Mundialización, comunicación y nuevo humanismo Implicaciones educativas”, en Agor@Digit@l, núm. 3. Disponible en: http://www. uhu.es/agora/version01/digital/numeros/03/03-articulos/monografico/pdf3/ medel.PDF [25 de enero de 2013].

Vázquez Medel, Manuel Ángel (2008/2009), “La semiótica de la cultura y la construcción 
del imaginario social", en Entretextos, 11-12-13. Disponible en: http://www.ugr. es/ mcaceres/entretextos/entre11-12/pdf/vazquez.pdf [25 de enero de 2013].

Waters, Richard (20 de noviembre de 2006), "Wikipedia stand-off in search for online truth", en Financial Times. Disponible en: http://www.ft.com/intl/cms/s/0/3300554a6d6a-11db-8725-0000779e2340.html [25 de enero de 2013].

Elisa Alonso Jiménez. Diploma de Estudios Avanzados en el Programa Interdisciplinar de Doctorado en Estudios Culturales (Universidad de Sevilla, España, 2010). Profesora e investigadora a tiempo completo en el Departamento de Filología y Traducción, Universidad Pablo de Olavide de Sevilla (España). Líneas de investigación: tecnologías de la traducción y la comunicación, estudios de traducción. Publicaciones recientes: Elisa Alonso Jiménez, "Nuevos recursos de investigación en torno a traducción, tecnología informática y español”, en E. Calvo Encinas, M. Enríquez Aranda, N. Jiménez Carra et al. [eds.], La traductología actual: nuevas vias de investigación en la disciplina (Colección Interlingua), Granada: Comares (2010); Elisa Alonso Jiménez, "Linguee y las nuevas formas de traducir", en Skopos. Revista Internacional de Traducción e Interpretación, núm. 2, Córdoba, España: Universidad de Córdoba (2013); Elisa Alonso Jiménez y Elisa Calvo Encinas, "Evolución de Paradigmas en Traductología a la Luz de los Fenómenos Tecnológicos", en N. A. Campos Plaza, E. Alarcón Navío y L. Herrero Rodes [eds.], Translating Culture-Traduire la Culture-Traducir la cultura. Politicas lingüisticas de comunicación internacional y traducción institucional (Colección Interlingua), Granada: Comares (2014).

Recepción: 28 de febrero de 2014 .

Aprobación: 9 de diciembre de 2014. 
